\title{
Decreased expression of TROAP suppresses cellular proliferation, migration and invasion in gastric cancer
}

\author{
KE JING, QINSHENG MAO and PENG MA \\ Department of Gastrointestinal Surgery, Affiliated Hospital of Nantong University, Nantong, Jiangsu 226001, P.R. China
}

Received December 29, 2016; Accepted November 11, 2017

DOI: $10.3892 / \mathrm{mmr} .2018 .9230$

\begin{abstract}
Trophinin associated protein (TROAP) is a cytoplasmic protein required for spindle assembly and cell invasion; however, its biological function in cancer remains to be elucidated. In the present study, by analyzing three independent datasets from the Oncomine database, it was identified that TROAP mRNA expression was upregulated in gastric cancer (GC) tissues compared with normal counterparts. Furthermore, elevated expression of TROAP was associated with poor survival in patients with GC, as predicted using Kaplan-Meier analysis. TROAP was knocked down to verify its functional role in gastric cancer cell lines, SGC-7901 and MGC80-3. MTT assay was used to analyze cell proliferation. Cell cycle progression, and migration and invasion were determined using flow cytometry and Transwell assay, respectively. In vitro experiments demonstrated that knockdown of TROAP significantly suppressed cell proliferation, G1 to S cell cycle transition, and the migration and invasion ability of GC cells. The results of the present study suggest that TROAP is overexpressed in GC and serves an oncogenic role in gastric cancer by affecting cell proliferation and invasion.
\end{abstract}

\section{Introduction}

Gastric cancer (GC) is the third most common malignant solid cancer and a global public health concern $(1,2)$. As in other cancers, GC is characterized by uncontrolled proliferation, extensive invasion and distant metastasis, primarily due to multiple risk factor-induced genetic alterations (3-5). Certain developments have been achieved in the diagnosis and therapy of GC over previous decades, but the long-term prognosis of patients with GC has not markedly improved (6). The primary

Correspondence to: Mr. Qinsheng Mao or Mr. Peng Ma, Department of Gastrointestinal Surgery, Affiliated Hospital of Nantong University, 20 Xisi Road, Nantong, Jiangsu 226001, P.R. China

E-mail: qsmao_1960@yeah.com

E-mail: mapengp_0809@163.com

Key words: gastric cancer, trophinin associated protein, cell cycle, invasion, cell proliferation reason for poor prognosis is late diagnosis at an advanced stage of the disease due to insufficient understanding of the mechanisms underlying GC (7). Therefore, it is necessary to improve early diagnosis and develop effective treatment for GC.

Trophinin associated protein (TROAP; also termed tastin) was first described as a soluble cytoplasmic protein that forms a complex with trophinin and bystin, and participates in early embryo implantation by mediating cellular invasion and proliferation (8-11). Human TROAP is composed of 778 amino acid residues rich in proline, with a basic $\mathrm{N}$ terminus and an acidic $\mathrm{C}$ terminus. TROAP is abundant in testes, bone marrow and thymus tissues (12). It contains five putative cyclin recognition sites that could target the mitotic kinase to appropriate substrates. A previous study has demonstrated that TROAP is essential for spindle assembly and monopolar spindle formation, which serve an important role in maintaining structural and dynamic features of centrosomes during mitosis (13). A microarray analysis based on genomics data from the Genomics Institute of the Novartis Research Foundation database demonstrated that TROAP is upregulated in human cancer cell lines, including HeLa and Jurkat. A previous study indicated that TROAP expression is also upregulated in prostate cancer tissues (14). The above data strongly suggest that increased TROAP expression may serve a role in GC proliferation and metastasis.

To validate this hypothesis, the expression pattern of TROAP was determined using the Oncomine database and GC cell lines. The effect of dysregulation of TROAP expression on patient overall survival was predicted using the Kaplan-Meier plotter. Furthermore, the role of TROAP in GC cell lines was investigated by analyzing cell proliferation, cell cycle distribution and invasive capability.

\section{Materials and methods}

Oncomine microarray database analysis. Expression of TROAP was retrieved from the Oncomine database (https://www.oncomine.org/resource/login.html). A combined filter was used to display corresponding datasets to determine the differential expression of TROAP between GC and their normal counterparts by defining the cancer type was GC, data type as mRNA and analysis type as cancer vs. normal analysis. The original data, including the Cui gastric (15), Cho gastric (16), Chen gastric (17) and Wang gastric (18) datasets were further analyzed and results visualized using the 
GraphPad Prism software (version 5.0; GraphPad Software, Inc., La Jolla, CA, USA) (15-18).

Kaplan-Meier overall survival analysis. The prognostic value of the TROAP gene in GC was analyzed using the Kaplan-Meier plotter (http://kmplot.com/analysis/). Samples have been classified as either, increased or lower expression levels compared with the median expression level of TROAP in GC tissues. Patient survival information was compared using a Kaplan-Meier survival plot. The hazard ratios with 95\% confidence intervals and log rank P-values were calculated using independent sample $t$ test. In order to reduce the false discovery rate, $\mathrm{P}<0.01$ was considered to indicate a statistically significant difference.

Cell lines and culture conditions. Human GC cell lines (AGS, SGC-7901, MGC80-3 and BGC-823) and a normal gastric mucosa epithelial cell line GES-1 were obtained from the American Type Culture Collection (Manassas, VA, USA). AGS cells were cultured in F12 medium (Sigma-Aldrich; Merck KGaA, Darmstadt, Germany) containing $10 \%$ fetal bovine serum (FBS; Gibco; Thermo Fisher Scientific, Inc., Waltham, MA, USA). The remaining cell lines were cultured in RPMI-1640 medium (HyClone; GE Healthcare Life Sciences, Logan, UT, USA) supplemented with $10 \%$ FBS (Gibco; Thermo Fisher Scientific, Inc.). All cell lines were incubated in a humidified atmosphere with $5 \% \mathrm{CO}_{2}$ at $37^{\circ} \mathrm{C}$.

Cell transfection. For the knockdown of TROAP (NCBI reference sequence NM_005480), two sequences targeting TROAP (TROAP-shRNA1: 5'-CCGGCCTCCAACTCTGAC CTCATATCTCGAGATATGAGGTCAGAGTTGGAGGTT TTTG-3' and TROAP-shRNA2: 5'-CCGGGCCCTGTGTTT CATTCCAGTTCTCGAGAACTGGAATGAAACACAGGG CTTTTTG-3'), and a scrambled negative control (NC) sequence (5'-TTCTCCGAACGTGTCACGT-3') were designed and synthesized by Sigma-Aldrich (Merck KGaA). These stem-loop-stem oligo small hairpin RNAs (shRNAs) were synthesized, annealed and ligated into a pLKO.1-TRC vector (Addgene, Inc., Cambridge, MA, USA) between the Agel and EcoRI sites. Ligation was confirmed by DNA sequencing (Shanghai Sangong Pharmaceutical Co., Ltd., Shanghai, China). Lentiviral particles were generated by transient transfection of 293 T cells (Cell Bank of Chinese Academy of Science, Shanghai, China) with TROAP-shRNA (or a scrambled sequence) plasmid (from Professor ZhangXu lab; Institute of Genetics and Developmental Biology, Chinese Academy of Sciences, Beijing, China) at a multiplicity of infection of 45 using Lipofectamine 2000 transfection reagent (Thermo Fisher Scientific, Inc.), according to the manufacturer's protocol. Lentivirus particles expressing TROAP-shRNA1, TROAP-shRNA2 or a scrambled sequence were named shTROAP-1, shTROAP-2 and NC, respectively. At $72 \mathrm{~h}$ after transfection, lentiviral particles were harvested by ultracentrifugation $\left(50,000 \mathrm{x} \mathrm{g}\right.$ at $4^{\circ} \mathrm{C}$ for $\left.15 \mathrm{~min}\right)$. For cell transfection, SGC-7901 and MGC80-3 cell lines were seeded in 24-well plates and transfected with constructed lentiviruses containing shTROAP-1, shTROAP-2 or NC, respectively. Subsequently, knockdown efficiency of TROAP was examined by western blot analysis 4 days after transfection.
Western blot analysis. Cells were lysed using a radioimmunoprecipitation assay lysis buffer (Beyotime Institute of Biotechnology, Haimen, China) supplemented with a Protease Inhibitor Cocktail (Sigma-Aldrich; Merck KGaA) for protein extraction. Protein concentration was determined by bicinchoninic acid protein assay kit (Beyotime Institute of Biotechnology). A total of $30 \mu \mathrm{g}$ protein samples were separated by $10 \%$ SDS-PAGE and transferred to polyvinylidene fluoride membranes (EMD Millipore, Billerica, MA, USA). Western blot analysis was performed by incubation in $5 \%$ skimmed milk blocking reagent at room temperature for $1 \mathrm{~h}$, followed by primary antibody against TROAP $(1: 1,000$; cat. no. ab14531, Abcam, Cambridge, UK) and GAPDH (1:50,000; cat. no. 10494-1-AP, ProteinTech Group, Inc., Chicago, IL, USA) at $4^{\circ} \mathrm{C}$ overnight. Membranes were washed with $\mathrm{NaCl} /$ Tris-Tween (Shanghai Sangong Pharmaceutical Co., Ltd.) and incubated with a horseradish peroxidase-conjugated secondary antibody (1:5,000; cat. no. SC-2054; Santa Cruz Biotechnology, Inc., Dallas, TX, USA) for $2 \mathrm{~h}$ at room temperature. The blots were detected using an enhanced chemiluminescence reagent (Amersham; GE Healthcare, Chicago, IL, USA). GAPDH was used as an internal control.

Cell proliferation assay. MTT assay (Sigma-Aldrich; Merck KGaA) was used to evaluate the proliferation rate of lentivirus-shRNA infected cells according to manufacturer's protocol. Cells were seeded into 96-well plates at a density of $1 \times 10^{4}$ cells/well and cultured in RPMI-1640 medium supplemented with $10 \%$ FBS. Cell proliferation was determined at 24,48 and $72 \mathrm{~h}$ by adding $20 \mu \mathrm{l}$ MTT $(5 \mathrm{mg} / \mathrm{ml})$ to each well, followed by incubation for $4 \mathrm{~h}$. The reaction was terminated by removal of the supernatant and addition of $100 \mu \mathrm{l}$ dimethyl sulfoxide (Sigma-Aldrich; Merck KGaA). The absorbance was measured at a wavelength of $490 \mathrm{~nm}$ using an ELISA microplate reader. Analysis of each sample was repeated three times.

Cell cycle analysis. Flow cytometry was used to analyze cell cycle in GC cells after 4 days after transfection. Briefly, cells were seeded into 6-well plates, harvested by trypsinization and fixed in ice-cold $70 \%$ ethanol overnight. Fixed cells were subsequently stained with $200 \mu$ l propidium iodide at $4^{\circ} \mathrm{C}$ for $30 \mathrm{~min}$. Cellular DNA content from each sample was determined using a BD FACSCaliber flow cytometer and ModFit LT software (Version 3.2; BD Biosciences, San Jose, CA, USA). Analysis of each sample was repeated three times.

Cell migration and invasion assays. Transwell assay was used to detect the migration and invasion of GC cells $96 \mathrm{~h}$ following transfection. For cell migration, cells $\left(1 \times 10^{5}\right.$ cells/well $)$ in $200 \mu \mathrm{l}$ serum-free medium were seeded in 24 -well plates with an 8-mm pore membrane insert (Corning Incorporated, Corning, NY, USA) in the upper chamber. A total of $500 \mu \mathrm{l}$ $10 \%$ FBS-containing medium was used as a chemoattractant in the lower chamber. After $24 \mathrm{~h}$ incubation, cells that migrated into the lower side of the membrane were stained with $0.1 \%$ crystal violet for $10 \mathrm{~min}$ at $37^{\circ} \mathrm{C}$. For the cell invasion assay, the procedure was similar to the cell migration assay, except that the upper chamber was pre-coated with Matrigel (Corning Incorporated). Stained 

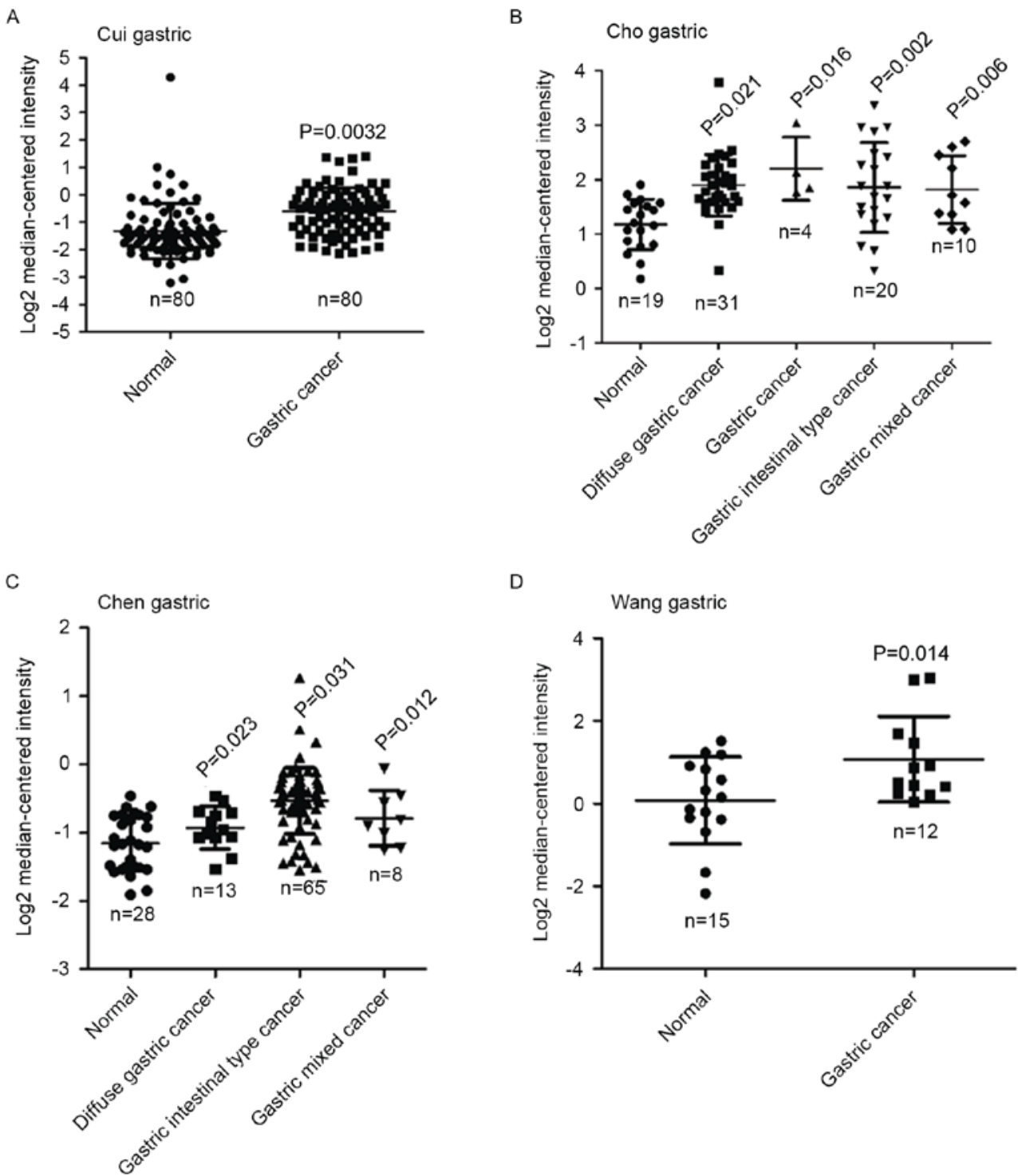

Figure 1. TROAP expression is upregulated in gastric cancer tissues. Analysis of TROAP expression in public Oncomine datasets, including (A) Cui gastric, (B) Cho gastric, (C) Chen gastric and (D) Wang gastric datasets. P-value vs. normal control tissues. TROAP, trophinin associated protein.

cells at the bottom of the membrane were observed and counted under a fluorescent microscope (Olympus $\mathrm{CH}-2$, Olympus Corporation, Tokyo, Japan). Experiments were performed three times.

Statistical analysis. All data were analyzed using SPSS software (version 18.0; SPSS, Inc., Chicago, IL, USA) and GraphPad Prism software (version 5.0) and expressed as the mean \pm standard deviation of three independent experiments. The statistical difference among groups was evaluated by one-way analysis of variance or two-tailed Student's t-test. $\mathrm{P}<0.05$ was considered to indicate a statistically significant difference.

\section{Results}

TROAP expression is upregulated in GC tissues. Using the Oncomine database, differential mRNA expression of TROAP was determined between GC tissues and normal gastric tissues. The results demonstrated that the expression of TROAP was significantly elevated in gastric cancer $(n=80$;
$\mathrm{P}=0.0032)$ compared with normal tissues $(\mathrm{n}=80)$ in the Cui gastric dataset (Fig. 1A). In the Cho gastric dataset, TROAP expression was significantly increased in diffuse gastric cancer $(\mathrm{n}=31 ; \mathrm{P}=0.021)$, gastric cancer $(\mathrm{n}=4 ; \mathrm{P}=0.016)$, gastric intestinal type cancer $(n=20 ; P=0.002)$ and gastric mixed cancer $(n=10 ; P=0.006)$ compared with normal tissues ( $n=19$; Fig. 1B). Similarly, TROAP expression was significantly increased in diffuse gastric cancer $(\mathrm{n}=13 ; \mathrm{P}=0.023)$, gastric intestinal type cancer $(n=65 ; P=0.031)$ and gastric mixed cancer $(n=8$; $\mathrm{P}=0.012$ ) in the Chen gastric datasets (Fig. 1C). Data derived from the Wang gastric dataset indicated that TROAP expression was also upregulated in gastric cancer $(n=15 ; P=0.014)$ compared with normal tissues ( $n=12$; Fig. 1D). The observations suggest that TROAP expression is dysregulated in GC.

Elevated expression of TROAP predicts poor survival in patients with $G C$. Furthermore, association between TROAP mRNA expression levels and overall survival in patients with GC was assessed using the Kaplan-Meier plotter (http://kmplot.com/analysis/). Elevated levels of 
TROAP expression were associated with lower survival rates in patients (hazard ratio $=1.89,95 \%$ confidence interval=1.57-2.27, $\mathrm{P}=5.1 \times 10^{-12}$; Fig. 2). The results demonstrated that TROAP may act as a prognostic oncogene for patients with GC.

Efficiency of shRNA knockdown of TROAP determined by western blot analysis. To further determine the potential oncogenic role of TROAP in GC, expression of TROAP was determined in several GC cell lines and a normal gastric cell line, GES-1. The expression level of TROAP protein was upregulated in all GC cells compared with GES-1 cells (Fig. 3A). SGC-7901 and MGC80-3 cells demonstrated markedly elevated TROAP expression. Therefore, both of SGC-7901 and MGC80-3 cell lines were cultured and transfected with NC or shTROAP. The expression levels of TROAP protein were decreased in shTROAP groups compared with NC groups in SGC-7901 and MGC80-3 cells (Fig. 3B and C, respectively). Notably, shTROAP-1 produced a stronger knockdown efficiency compared with shTROAP-2 in both cells and therefore shTROAP-1 was selected for the subsequent loss-of-function assays.

TROAP knockdown suppresses cell proliferation and cell cycle progression in GC. Proliferation rates of SGC-7901 and MGC80-3 cells transfected with shTROAP-1 were significantly reduced compared with the respective $\mathrm{NC}$ groups on the $72 \mathrm{~h}$ of the experiment ( $\mathrm{P}<0.001$; Fig. $4 \mathrm{~A}$ and $\mathrm{B})$. To determine the mechanism underlying the inhibition of cell growth, cell cycle distribution was detected in SGC-7901 and MGC80-3 cells following lentiviral transfection. The number of cells in the G0/G1 phase was significantly increased, while the number of cells in the G2/M phase was decreased in SGC-7901 cells transfected with shTROAP-1 compared with the NC group $(\mathrm{P}<0.05 ;$ Fig. $4 \mathrm{C})$. Similar results were observed in MGC80-3 cells treated with shTROAP-1 (Fig. 4D). These results indicated that the cell cycle was arrested in the G0/G1 phase following TROAP knockdown.

TROAP knockdown inhibits cell migration and invasion capacity in GC. Transwell assay was used to detect the effect of TROAP knockdown on cell migration and invasion. The number of cells that migrated to the lower membrane in the SGC-7901/shTROAP-1 and MGC80-3/shTROAP-1 groups was decreased markedly compared with the respective NC groups $(\mathrm{P}<0.05$; Fig. $5 \mathrm{~A})$. In addition, TROAP knockdown significantly impaired the invasion ability of SGC-7901 and MGC80-3 cells ( $\mathrm{P}<0.05$; Fig. 5A). As expected, knockdown of TROAP was associated with reduced migration and invasion abilities of GC cells.

\section{Discussion}

The role of TROAP in malignancy has attracted the interest of various research groups. TROAP, a soluble cytoplasmic protein, was reported to be overexpressed in HeLa and Jurkat cells and prostate cancer tissues, suggesting that TROAP is a tumor-associated gene (14). In the present study, mRNA expression of TROAP was significantly upregulated in GC tissues compared with normal tissues and upregulation of

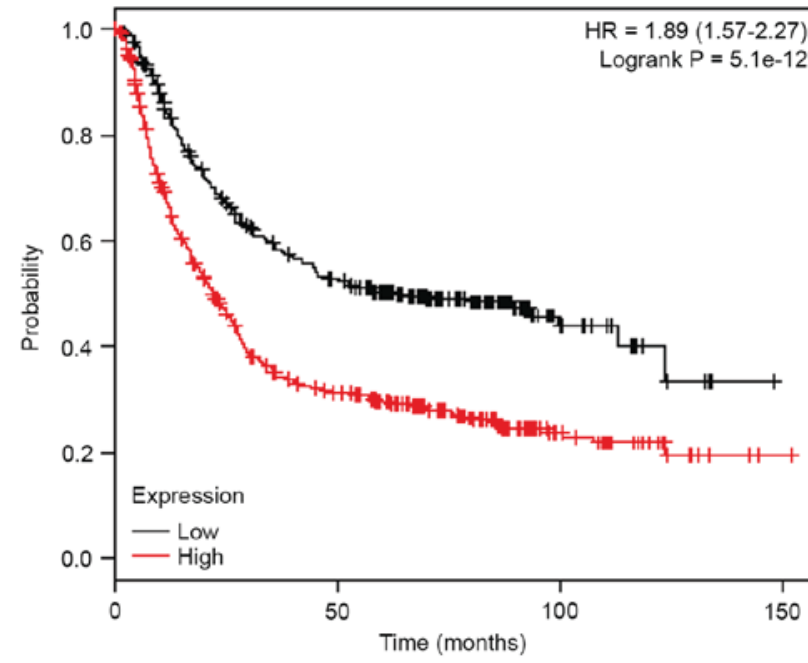

Number at risk

$\begin{array}{lrrr}\text { Low } 342 & 155 & 22 & 0 \\ \text { High } 534 & 143 & 26 & 1\end{array}$

Figure 2. Kaplan-Meier plots predicting overall survival in patients with gastric cancer dependent on TROAP expression. Red: Patients with TROAP expression above the median level. Black: Patients with TROAP expression below the median level. TROAP, trophinin associated protein; HR, hazard ratio.

A

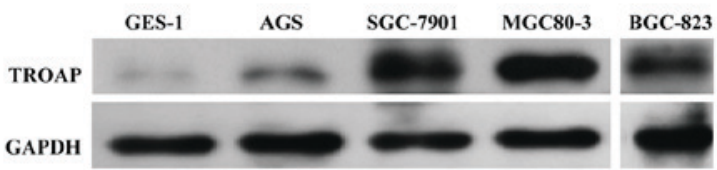

B

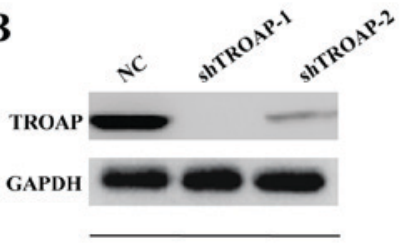

SGC-7901

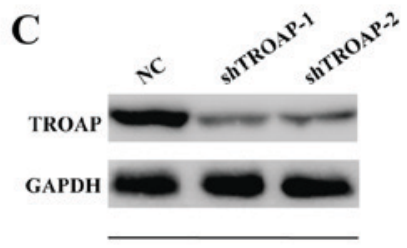

MGC80-3
Figure 3. Increased expression of TROAP was efficiently suppressed in GC cells. Western blot analysis of (A) TROAP in four different GC cell lines. Western blotting of TROAP in (B) SGC-7901 and (C) MGC80-3 cells following transfection with shTROAPs. GAPDH was used as internal control. $\mathrm{GC}$, gastric cancer; NC, negative control; TROAP, trophinin associated protein; sh, small hairpin.

TROAP was associated with lower survival rates predicted using an analysis of public online databases. In addition, the effect of TROAP on the biological behavior of GC cells was investigated. The results of the present study demonstrated that TROAP may have an oncogenic effect on GC.

Deregulated growth is a primary requirement for cancer development (19) and is closely associated with dysregulation of the cell cycle, which is composed of distinct sequential phases (G0/G1, S and G2/M) (19-21). TROAP is a cycling protein essential for cell cycle progression and its endogenous levels are tightly controlled during mitosis. Furthermore, TROAP contains multiple potential sites for serine/threonine phosphorylation by highly active protein 
A
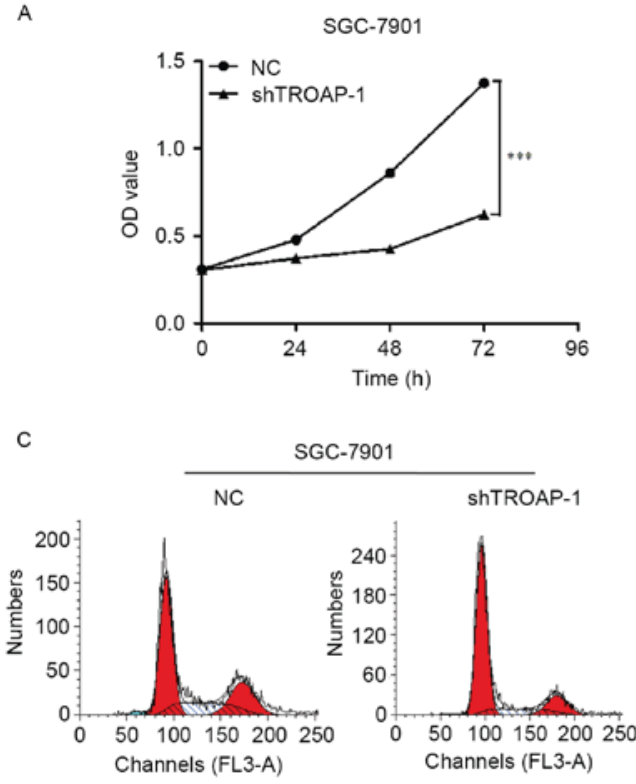
SGC-7901

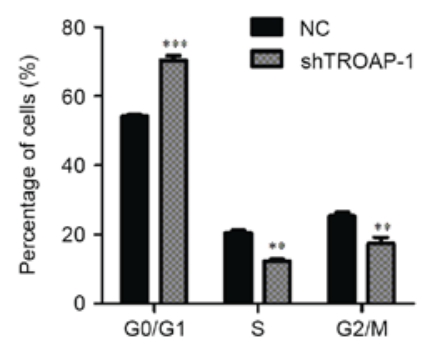

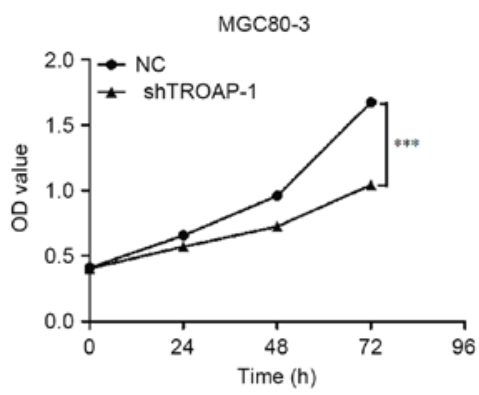

D

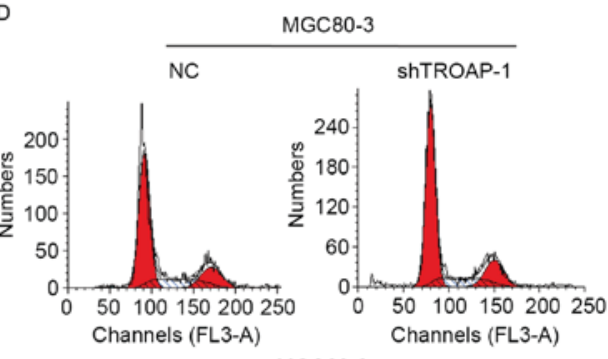

MGC80-3

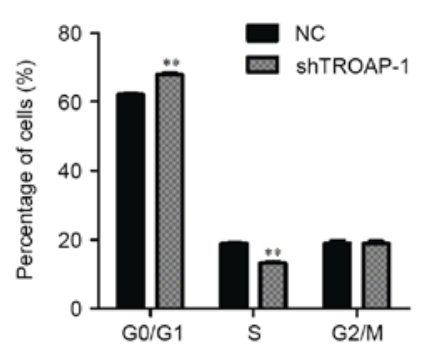

Figure 4. Knockdown of TROAP suppressed cell proliferation and induced cell cycle arrest in gastric cancer. MTT assay was used to analyze the proliferation of (A) SGC-7901 and (B) MGC80-3 cells following transfection with shTROAP-1. ${ }^{* * *} \mathrm{P}<0.001$. The number of (C) SGC-7901 and (D) MGC80-3 cells in different phases were determined by flow cytometry following transfection with shTROAP-1. Data are expressed as the mean \pm standard deviation of 3 independent experiments. ${ }^{* *} \mathrm{P}<0.01,{ }^{* * * *} \mathrm{P}<0.001$ vs. NC group. NC, negative control; sh, small hairpin; OD, optical density; TROAP, trophinin associated protein.

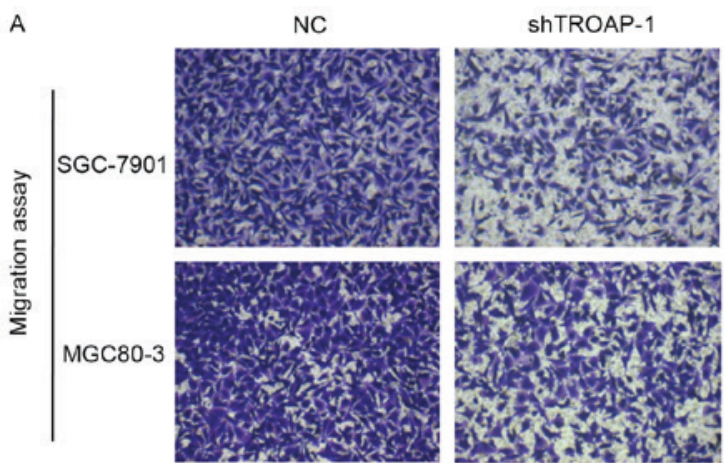

B

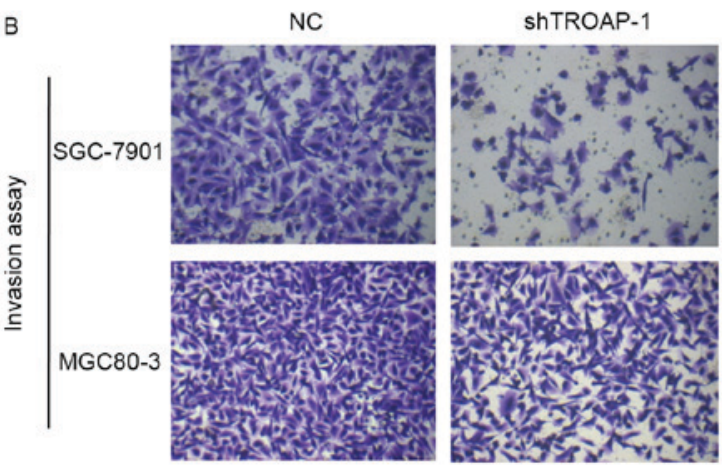

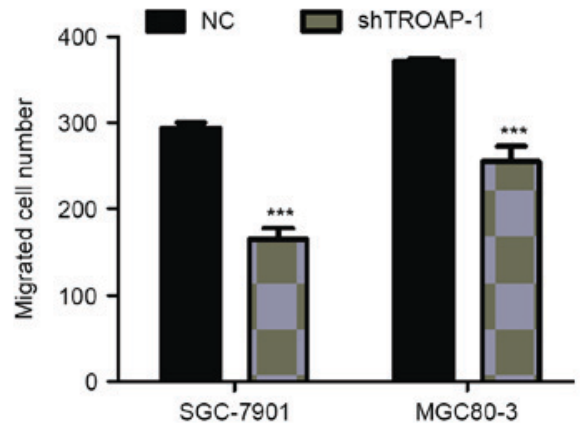

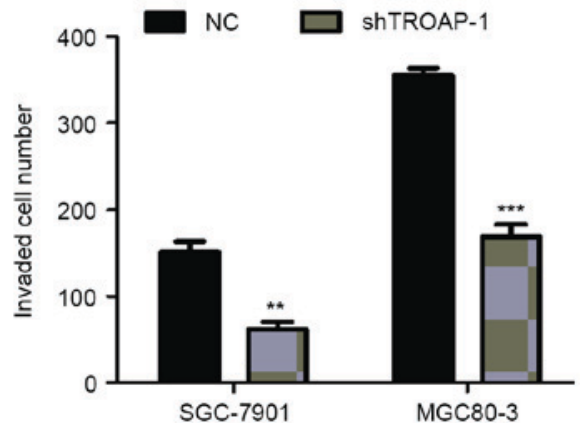

Figure 5. Effect of TROAP on gastric cancer cell migration and invasion. Effect of TROAP knockdown on (A) migration of SGC-7901 and MGC80-3 cells and (B) the invasive potential of SGC-7901 and MGC80-3 cells, as detected by Transwell assay. Data are expressed as the mean \pm standard deviation of three independent experiments. ${ }^{* *} \mathrm{P}<0.01,{ }^{* * *} \mathrm{P}<0.001$ vs. NC. NC, negative control; sh, small hairpin; TROAP, trophinin associated protein. 
kinases, including cyclin-dependent kinase 1, polo-like kinase and mitogen-activated protein kinases during mitosis (20). Consistent with the above evidence, in the present study reduced expression of TROAP by shRNA significantly suppressed cell proliferation of SGC-7901 and MGC80-3 by inhibiting G1 to $\mathrm{S}$ cell cycle transition. Resulting abnormal spindle assembly caused by overexpression of TROAP may in theory lead to genomic imbalances and contribute to oncogenesis $(21,22)$. The above data indicate that TROAP may contribute to gastric carcinogenesis.

In addition to cell proliferation, invasion is also a key step associated with the progression of tumor cells in target microenvironments. Therefore, a Transwell migration/invasion assay was performed to investigate the role of TROAP in GC cell motility. The results of the present study indicated that reduced expression of TROAP markedly decreased the migration and invasion ability of GC cell lines (SGC-7901 and MGC80-3). Previous studies demonstrated that TROAP and two other cytoplasmic proteins, trophinin and bystin are the components of an adhesion molecule complex required for the initial attachment of an embryo to the uterus $(11,23)$. Furthermore, overexpression of TROAP has been reported to promote gallbladder cancer invasion and metastasis (24). TROAP is necessary for the cell adhesion function of trophinin as it creates sites for efficient adhesion to the cell surface (9). TROAP act as a promoter of tumor migration and invasion during GC progression.

In conclusion, the present study provided evidence that TROAP serves a role in GC cells by promoting cell proliferation, cell cycle progression and invasion in vitro. Combined analysis of cell lines and datasets from a public database, demonstrated that TROAP overexpression may be used as a predictor of poor survival in patients with GC. The molecular mechanisms of TROAP in GC remain to be elucidated. The present study may be used for future application of TROAP-targeted therapy in preclinical and clinical studies of gastric cancer treatment.

\section{Acknowledgements}

Not applicable.

\section{Funding}

No funding was received.

\section{Availability of data and materials}

All data generated or analyzed during this study are included in this published article.

\section{Authors' contributions}

$\mathrm{KJ}$ and PM conceived and designed the experiments, and wrote the manuscript. KJ, QM and PM performed the experiments and analyzed the data.

\section{Ethics approval and consent to participate}

Not applicable.

\section{Consent for publication}

Not applicable.

\section{Competing interests}

The authors declare that they have no competing interests.

\section{References}

1. Ajani JA, Bentrem DJ, Besh S, D'Amico TA, Das P, Denlinger C, Fakih MG, Fuchs CS, Gerdes H, Glasgow RE, et al: Gastric cancer, version 2.2013: Featured updates to the NCCN guidelines. J Natl Compr Canc Netw 11: 531-546, 2013.

2. Chen W, Zheng R, Zeng H, Zhang S and He J: Annual report on status of cancer in China, 2011. Chin J Cancer Res 27: 2-12, 2015.

3. Chen DL, Ju HQ, Lu YX, Chen LZ, Zeng ZL, Zhang DS, Luo HY, Wang F, Qiu MZ, Wang DS, et al: Long non-coding RNA XIST regulates gastric cancer progression by acting as a molecular sponge of miR-101 to modulate EZH2 expression. J Exp Clin Cancer Res 35: 142, 2016.

4. Cai Y, Yi M, Chen D, Liu J, Guleng B, Ren J and Shi H: Trefoil factor family 2 expression inhibits gastric cancer cell growth and invasion in vitro via interactions with the transcription factor Sp3. Int J Mol Med 38: 1474-1480, 2016.

5. Lynch HT, Grady W, Suriano G and Huntsman D: Gastric cancer: New genetic developments. J Surg Oncol 90: 114-133, 2005.

6. Ju J, Wang N, Wang X and Chen F: A novel all-trans retinoic acid derivative inhibits proliferation and induces differentiation of human gastric carcinoma xenografts via up-regulating retinoic acid receptor $\beta$. Am J Transl Res 7: 856-865, 2015.

7. Yu B, Lv X, Su L, Li J, Yu Y, Gu Q, Yan M, Zhu Z1 and Liu B: MiR-148a Functions as a tumor suppressor by targeting CCK-BR via inactivating STAT3 and Akt in human gastric cancer. PLoS One 11: e0158961, 2016.

8. Fukuda MN, Sato T, Nakayama J, Klier G, Mikami M, Aoki D and Nozawa S: Trophinin and tastin, a novel cell adhesion molecule complex with potential involvement in embryo implantation. Genes Dev 9: 1199-1210, 1995.

9. Suzuki N, Zara J, Sato T, Ong E, Bakhiet N, Oshima RG, Watson KL and Fukuda MN: A cytoplasmic protein, bystin, interacts with trophinin, tastin, and cytokeratin and may be involved in trophinin-mediated cell adhesion between trophoblast and endometrial epithelial cells. Proc Natl Acad Sci USA 95: 5027-5032, 1998.

10. Fukuda MN and Nozawa S: Trophinin, tastin, and bystin: A complex mediating unique attachment between trophoblastic and endometrial epithelial cells at their respective apical cell membranes. Semin Reprod Endocrinol 17: 229-234, 1999.

11. Suzuki N, Nakayama J, Shih IM, Aoki D, Nozawa S and Fukuda MN: Expression of trophinin, tastin, and bystin by trophoblast and endometrial cells in human placenta. Biol Reprod 60: 621-627, 1999.

12. Nadano D, Nakayama J, Matsuzawa S, Sato TA, Matsuda T and Fukuda MN: Human tastin, a proline-rich cytoplasmic protein, associates with the microtubular cytoskeleton. Biochem J 364: 669-677, 2002

13. Yang S, Liu X, Yin Y, Fukuda MN and Zhou J: Tastin is required for bipolar spindle assembly and centrosome integrity during mitosis. FASEB J 22: 1960-1972, 2008.

14. Dhanasekaran SM, Barrette TR, Ghosh D, Shah R, Varambally S, Kurachi K, Pienta KJ, Rubin MA and Chinnaiyan AM: Delineation of prognostic biomarkers in prostate cancer. Nature 412: 822-826, 2001.

15. Cui J, Chen Y, Chou WC, Sun L, Chen L, Suo J, Ni Z, Zhang M, Kong X, Hoffman LL, et al: An integrated transcriptomic and computational analysis for biomarker identification in gastric cancer. Nucleic Acids Res 39: 1197-1207, 2011.

16. Cho JY, Lim JY, Cheong JH, Park YY, Yoon SL, Kim SM, Kim SB, Kim H, Hong SW, Park YN, et al: Gene expression signature-based prognostic risk score in gastric cancer. Clin Cancer Res 17: 1850-1857, 2011. 
17. Chen X, Leung SY, Yuen ST, Chu KM, Ji J, Li R, Chan AS, Law S, Troyanskaya OG, Wong J, et al: Variation in gene expression patterns in human gastric cancers. Mol Biol Cell 14: 3208-3215, 2003.

18. Wang Q, Wen YG, Li DP, Xia J, Zhou CZ, Yan DW, Tang HM and Peng ZH: Upregulated INHBA expression is associated with poor survival in gastric cancer. Med Oncol 29: 77-83, 2012.

19. Vermeulen K, Van Bockstaele DR and Berneman ZN: The cell cycle: A review of regulation, deregulation and therapeutic targets in cancer. Cell Prolif 36: 131-149, 2003.

20. Mayor T, Meraldi P, Stierhof YD, Nigg EA and Fry AM: Protein kinases in control of the centrosome cycle. FEBS Lett 452: 92-95, 1999.
21. Brinkley BR: Managing the centrosome numbers game: From chaos to stability in cancer cell division. Trends Cell Biol 11: 18-21, 2001.

22. Doxsey SJ: Centrosomes as command centres for cellular control. Nat Cell Biol 3: E105-E108, 2001.

23. Ayala GE, Dai H, Li R, Ittmann M, Thompson TC, Rowley D and Wheeler TM: Bystin in perineural invasion of prostate cancer. Prostate 66: 266-272, 2006

24. Chang XZ, Yu J, Zhang XH, Yin J, Wang T and Cao XC: Enhanced expression of trophinin promotes invasive and metastatic potential of human gallbladder cancer cells. J Cancer Res Clin Oncol 135: 581-590, 2009. 